\title{
Modelo Matemático para Simulação da Propagação Acústica Submarina e Seus Possíveis Impactos em Algumas Espécies de Cetáceos
}

\section{Mathematical Model for Simulation of Underwater Acoustic Propagation and its Possible Impacts on Some Species of Cetaceans}

Fabiano Marcos de Lima ${ }^{1}$, Claudia Mazza Dias ², Carlos Andrés Reyna VeraTudela ${ }^{3}$

\section{RESUMO}

Uma das características importantes dos mamíferos marinhos é sua capacidade de transmitir e recepcionar sons em ambiente submarino. Tal capacidade é fundamental para a sobrevivência desses mamíferos em seu habitat natural, fazendo com que esses animais sejam extremamente dependentes da paisagem acústica local. Atividades como comunicação, caça, fuga de predadores, orientação geográfica, acasalamento, entre outras, estão diretamente ligadas à capacidade desses animais de emitir e receber sons ao seu redor. A poluição sonora causada pelas ações humanas na execução de suas atividades produtivas nos mares e oceanos, pode interferir de uma forma negativa na rotina desses animais. O presente trabalho trata de um modelo matemático e computacional, baseado na equação diferencial parcial da onda, capaz de simular a intensidade da propagação acústica em um ambiente submarino. Desta forma, é possível gerar um panorama da poluição de ruídos gerados pelas ações humanas e relacioná-las com as características acústicas de algumas espécies de mamíferos marinhos, avaliando os possíveis impactos na vida desses animais.

Palavras-chave: Modelagem Matemática. Propagação Acústica. Mamíferos Marinhos. Cetáceos. Poluição Sonora.

\section{ABSTRACT}

One of the important characteristics of marine mammals is their ability to transmit and receive sounds in an underwater environment. Such ability is fundamental to the survival of these mammals in their natural habitat, making these animals extremely dependent on the local acoustic landscape. Activities such as communication, hunting, predator flight, geographic orientation, mating, among others, are directly linked to the ability of these animals to emit and receive sounds around them. The noise pollution caused by human actions in the execution of their productive activities in the seas and oceans, can interfere in a negative way in the routine of these animals. The present work deals with a mathematical and computational model, based on the partial differential equation of the wave, able to simulate the intensity of the acoustic propagation in an underwater environment. In this way, it is possible to generate an overview of the pollution of noises generated by human actions and to relate them to the acoustic characteristics of some species of marine mammals, evaluating the possible impacts on the lives of these animals.

Keywords: Mathematical Modeling. Acoustic Propagation. Marine Mammals. Cetaceans. Noise pollution.

1 Mestre em Modelagem Matemática Computacional.

E-mail:

fabianomarcos1@hotmail. com

2 Doutora em Engenharia Civil e Professora do

Programa de Pós-

graduação em Modelagem Matemática e

Computacional da UFRRJ.

E-mail:

mazzaclaudia@gmail.com

3 Doutor em Engenharia Civil e Professor do Programa de Pósgraduação em Modelagem Matemática Computacional da UFRRJ.

\section{E-mail:}

carlos.reyna.veratudela@ gmail.com 


\section{INTRODUÇÃO}

Os ruídos subaquáticos, em especial os gerados pelas atividades antropogênicas (produzidas pelo homem) oriundos do trânsito de navios, embarcações entre outros veículos, que podem irradiar ruídos para os oceanos, vêm causando uma crescente preocupação com os potenciais impactos no meio ambiente e no ecossistema marinho em locais com intensa atividade humana e são considerados especialmente perigosos para os mamíferos marinhos (RICHARDSON \& WÜRSIG, 1995; dos SANTOS et al., 2010).

Atualmente, em nosso país, observa-se a crescente demanda das atividades econômica nos oceanos e a necessidade de realizar trabalhos de pesquisa e mapeamento geográfico, transporte de passageiros, escoamento de óleo e gás, entre outras atividades relacionadas. Nota-se o aumento significativo do tráfego de navios e embarcações, principalmente em regiões de descoberta de poços de petróleo, onde antes não havia presença massiva de máquinas e demais atividades antropogênicas (JESUS et al., 2010).

A busca de melhores formas de compreensão da propagação do som nos oceanos, em especial dos ruídos causados pelas ações antropogênicas, é de extrema importância para que estes afetem minimamente a vida marinha local. Sabe-se que a medição do nível de ruído nos oceanos é uma atividade que demanda muitos recursos financeiros e que muitas vezes não estão disponíveis para tal. Uma forma de contornar essa situação e minimizar o custo dos estudos para o controle da poluição sonora nos oceanos, é através da modelagem matemática e computacional, que pode prover ferramentas capazes de simular a propagação sonora, e assim ajudar a compreender melhor esse fenômeno, contribuindo no controle da poluição sonora submarina, e consequentemente, preservando o bem-estar dos animais marinhos que dependem do som como meio de vida.

A modelagem matemática vem sendo cada vez mais utilizada graças a sua ampla gama de possibilidades e aplicações, tornando-se uma ferramenta eficaz para a simulação de problemas reais, qual é o caso do modelo aqui proposto.

Em geral, navios possuem característica vibratória, que tem origem a partir do funcionamento de sua hélice e demais equipamentos que estão a bordo. Essas fontes de ruídos são classificadas como fontes contínuas e permanentes (REDONDO \& MATEO, 
2017). Os ruídos subaquáticos, gerados por atividades antropogênicas, como a operação de navios, é uma crescente preocupação com os potenciais impactos e danos que podem causar no ecossistema e principalmente na vida dos mamíferos marinhos que habitam regiões de intensas atividades humanas (ERBE, 2011). Assim, este trabalho tem como objetivo aplicar técnicas de modelagem matemática e computacional, com base na equação da onda, para simular a propagação de ruídos considerando um ambiente aquático para analisar os possíveis impactos na vida de baleias (Misticetos).

\section{EQUAÇÃO DA ONDA E PROPAGAC̣ÃO ACUSTICA}

A equação da onda é a equação diferencial parcial que modela fenômenos físicos onde ocorrem variações de grandezas de forma vibratória. Essas variações são causadas por uma perturbação que, a partir de uma condição inicial, modificam o equilíbrio de um determinado meio ou material. A propagação do som é um exemplo desse tipo de variação, por se tratar de uma onda mecânica capaz de se propagar em um ambiente subaquático.

Nos oceanos, os sons são produzidos basicamente pelas atividades ambientais, ou seja, ruídos gerados por algum fator da natureza como sísmicas, movimentos das marés, chuvas, entre outros. Os ruídos são gerados também pelas atividades dos animais marinhos como comunicação submarina, caça, mastigação, deslocamento, entre outras e pela ação do homem, que são ruídos gerados pelas atividades de produção humana como pesca e atividades de produção industrial, por exemplo (JENSEN et al., 2011). A equação que modela a propagação acústica é a equação da onda, definida como (IÓRIO, 2005):

$$
\nabla^{2} p-\frac{1}{c^{2}} \frac{\partial^{2} p}{\partial t^{2}}=f(t)
$$

onde $\nabla^{2}$ é o operador Laplaciano, $p$ é a pressão acústica, $c$ a velocidade do som, $t$ é o tempo de propagação e $f(t)$ uma função da fonte acústica, definida por:

$$
f(t)=B+A \operatorname{sen}(\omega t)
$$

onde $B$ é a posição do sinal acústico em relação ao eixos das abscissas, $A$ é a amplitude do sinal, e $\omega$ é a frequência angular em rad/s. Quando o sinal é composto por mais de 
uma frequência e amplitudes diferentes, a equação (2) pode representar um sinal com seis frequências com suas respectivas amplitudes:

$$
\begin{gathered}
f(t)=B+A_{1} \operatorname{sen}\left(\omega_{1} t\right)+A_{2} \operatorname{sen}\left(\omega_{2} t\right)+A_{3} \operatorname{sen}\left(\omega_{3} t\right)+A_{4} \operatorname{sen}\left(\omega_{4} t\right)+A_{5} \operatorname{sen}\left(\omega_{5} t\right) \\
+A_{6} \operatorname{sen}\left(\omega_{6} t\right)
\end{gathered}
$$

O nível de intensidade ou IL (Intensity Level) do som, é uma medida dada em termos da potência sonora. A potência é definida pelo trabalho em função do tempo e sua unidade no SI (Sistema Internacional) é dada em Watts (equivalente a $1 \mathrm{~J} / \mathrm{s}$ ). Em acústica submarina, ao se medir a intensidade do som, utiliza-se Pascal como unidade de referência da pressão acústica, sendo que $\mu P a$ equivale a $1 \mathrm{~N} / \mathrm{m}$. Desta forma, a intensidade sonora em decibéis $(\mathrm{dB})$ é estabelecida em termos do nível de pressão sonora ou SPL (Sound Level Pressure) em uma determinada área, da seguinte forma (AU \& HASTINGS, 2008):

$$
S P L=20 \log \left(\frac{p}{p_{r e}}\right)(d B)
$$

onde $p_{r e}$ é a pressão de referência, dada em $\mu P a$.

Os níveis das fontes sonoras utilizados neste trabalho foram obtidos a partir do estudo de McKenna e coautores (MCKENNA et al., 2012), que descreve e compara a emissão de energia acústica considerando as características espectrais de sete classes de navios de produção, enquanto em suas atividades normais de operação. Os níveis de potência acústica e frequências destes navios podem ser observados na Tabela 1, adaptada do trabalho dos mesmos autores.

Tabela 1. Níveis de Potência Acústica e Frequências de Navios - Adaptado de McKenna et al. (2012).

\begin{tabular}{l|cccccc}
\hline \multirow{2}{*}{ Classe de Navio } & \multicolumn{5}{c}{ Frequência - banda de uma-oitava $(\mathrm{Hz})$} \\
\cline { 2 - 7 } & $\mathrm{A}_{1}$ & $\mathrm{~A}_{2}$ & $\mathrm{~A}_{3}$ & $\mathrm{~A}_{4}$ & $\mathrm{~A}_{5}$ & $\mathrm{~A}_{6}$ \\
\hline Navio-tanque Químico & 175 & 175 & 174 & 173 & 174 & 168 \\
Navio-tanque Óleo Cru & 174 & 175 & 175 & 172 & 171 & 168 \\
Navio-tanque de Produtos & 174 & 176 & 175 & 169 & 170 & 165 \\
Navio de Carga & 168 & 177 & 175 & 172 & 173 & 168 \\
Navio Graneleiro & 170 & 177 & 183 & 176 & 174 & 170 \\
Navio Portardor & 172 & 173 & 173 & 171 & 174 & 168 \\
Navio Container & 179 & 178 & 177 & 177 & 178 & 174 \\
\hline
\end{tabular}


A partir dos valores dos níveis de pressão acústica apresentados na Tabela 1, é possível determinar as amplitudes das ondas que compõem o sinal acústico de cada classe de navio aqui considerada, dado importante na modelagem do problema.

\section{OS CETÁCEOS}

O termo mamífero marinho é utilizado para representar todas as espécies de animais mamíferos que vivem, a maior parte ou todo o seu tempo, em um habitat marinho, sendo a maioria das espécies pertencentes às águas salgadas e algumas outras às águas doces. Os mamíferos marinhos formam 4 ordens de animais, das quais três ordens são exclusivamente aquáticas: a ordem dos Cetáceos, que é dividida nas subordens Misticetos e Odontocetos, ordem Sirenia ou Seacows e os Pinípedes ou Selos (ordem Pinnipedia). A quarta ordem, carnívora, embora principalmente terrestre, inclui um considerável número de mamíferos marinhos, como a lontra-marinha (Enhydra Lutris) e o urso polar (Ursus Maritimus) (JEFFERSON et al., 2015).

A ordem dos Cetáceos é muito conhecida pelas baleias, golfinhos e botos, mamíferos marinhos muito encontrados em águas salgadas, e doces no caso dos botos. São conhecidas cerca de 80 espécies em todo o mundo, divididas em duas subordens: Misticetos e Odontocetos. Os Cetáceos são geralmente encontrados em águas costeiras e estuarinas a uma profundidade de aproximadamente 50 metros. Em águas mais profundas já consideradas offshore é possível encontrá-los em profundidades superiores a 200 metros (WALSH \& KOBAYASHI, 2004).

Embora toda ordem dos Cetáceos se utilize do som como meio de vida, este trabalho irá abordar apenas uma das subordens, a dos Misticetos, uma vez que estes emitem suas vocalizações em baixas frequências e com isso são mais sensíveis às características dos ruídos gerados pelos navios (AU \& HASTINGS, 2008).

Quando os mamíferos marinhos são expostos a altos níveis de intensidade sonora por um determinado tempo, as células capilares da cóclea de seu sistema auditivo, começam a fatigar podendo causar perdas auditivas temporárias ou permanentes. Se o animal for frequentemente submetido a altos níveis de ruídos, com o passar do tempo, sua sensibilidade auditiva vai diminuindo gradativamente à medida que é exposto a tais condições. Se os níveis de ruídos estiverem em um limiar próximo ou abaixo de um determinado limite crítico de energia sonora, as células capilares sofrem deformações 
DOI: $10.18605 / 2175-7275 /$ cereus.v12n2p104-118 Revista Cereus

2020 Vol. 12. N.2
DE LIMA, F.M. DIAS, C.M. TUDELA, C.A.R.V

Modelo Matemático para Simulação da Propagação Acústica

Submarina e Seus Possíveis Impactos em Algumas Espécies de Cetáceos

mas acabam retornando à sua forma normal causando um efeito de perda auditiva temporária (AU \& HASTING, 2008). Esse efeito é denominado Temporary Threshold Shift - TTS. Se esses animais forem expostos constantemente a níveis de intensidade sonora que excedam um determinado limite crítico, com o passar do tempo as células capilares podem ficar permanentemente danificadas e ele acabará morrendo. Esse efeito é denominado Permanent Threshold Shift - PTS. Os limites críticos da intensidade sonora que podem causar o efeito TTS variam entre 179 a $201 d B$ (AU \& HASTING, 2008).

As vocalizações das baleias podem ser divididas basicamente em duas categorias: cantos e chamadas. As chamadas podem ser subdivididas em outras três categorias como: chamadas simples, chamadas complexas e outras chamadas, que são compostas por cliques, pulsos, batidas e grunhidos. A Tabela 2 mostra um resumo da vocalização de algumas espécies de baleias com ênfase nas propriedades acústicas das chamadas.

Tabela 2. Frequências de Vocalização de Algumas Espécies de Baleias - Adaptada de AU \& HASTING (2008).

\begin{tabular}{|c|c|c|c|c|}
\hline Espécie de Baleia & Tipo de Sinal & $\begin{array}{c}\text { Limites de } \\
\text { Frequência } \\
(\mathrm{Hz})\end{array}$ & $\begin{array}{c}\text { Frequência } \\
\text { Dominante } \\
(\mathrm{Hz})\end{array}$ & $\begin{array}{l}\text { Nível Sonoro } \\
\text { (dB) }\end{array}$ \\
\hline Baleia Azul & $\begin{array}{l}\text { Gemidos FM } \\
\text { Cantos }\end{array}$ & $\begin{array}{c}11.5-200 \\
16-60\end{array}$ & $\begin{array}{l}16-25 \\
16-60\end{array}$ & 188 \\
\hline $\begin{array}{c}\text { Baleia Franca da } \\
\text { Groelândia }\end{array}$ & $\begin{array}{c}\text { Gemidos } \\
\text { Pulsos } \\
\text { Cantos }\end{array}$ & $\begin{array}{l}25-900 \\
25-350 \\
20-500 \\
\end{array}$ & $\begin{array}{l}100-400 \\
152-185 \\
158-189\end{array}$ & $129-178$ \\
\hline Baleia Bryde & $\begin{array}{c}\text { Gemidos } \\
\text { Gemidos em } \\
\text { Pulsos } \\
\text { Pulsos Discretos } \\
\end{array}$ & $\begin{array}{l}70-245 \\
100-930 \\
700-950 \\
\end{array}$ & $\begin{array}{l}124-132 \\
165-900 \\
700-950 \\
\end{array}$ & $152-174$ \\
\hline Baleia Fin & $\begin{array}{c}\text { Gemidos FM } \\
\text { Tonal } \\
\text { Cantos } \\
\end{array}$ & $\begin{array}{c}14-118 \\
34-150 \\
17-25 \\
\end{array}$ & $\begin{array}{c}20 \\
34-150 \\
17-25 \\
\end{array}$ & $\begin{array}{c}160-186 \\
186 \\
\end{array}$ \\
\hline Baleia Cinza & $\begin{array}{c}\text { Pulsos } \\
\text { Gemidos FM } \\
\text { Gemidos LF-PM } \\
\text { Pulsos PM }\end{array}$ & $\begin{array}{c}100-2000 \\
250-300 \\
125-1250 \\
150-1570\end{array}$ & $\begin{array}{c}300-825 \\
250-300 \\
<430 \\
225-600\end{array}$ & 152 \\
\hline Baleia Jubarte & $\begin{array}{c}\text { Gemidos Simples } \\
\text { Gemidos } \\
\text { Complexo } \\
\text { Pulsos } \\
\text { Cantos } \\
\text { Cliques }\end{array}$ & $\begin{array}{c}20-1800 \\
35-360 \\
25-89 \\
30-8000 \\
500-2000\end{array}$ & $\begin{array}{c}25-30 \\
35-360 \\
25-80 \\
120-4000 \\
800,1800\end{array}$ & $\begin{array}{c}175 \\
175 \\
176 \\
144-174 \\
143-154\end{array}$ \\
\hline Baleia Minke & $\begin{array}{c}\text { Tonal FM } \\
\text { Batidas } \\
\text { Grunhidos }\end{array}$ & $\begin{array}{c}60-130 \\
100-200 \\
60-140\end{array}$ & $\begin{array}{c}60-130 \\
100-200 \\
60-140\end{array}$ & $\begin{array}{c}165 \\
151-175\end{array}$ \\
\hline
\end{tabular}




\section{MATERIAIS E METODOS}

O modelo matemático da propagação acústica em ambiente submarino, proposto para este trabalho, consiste na simulação do campo acústico gerado por uma ou mais fontes emitindo sinais que podem ser sensíveis aos mamíferos marinhos da subordem dos Misticetos que trafegam nas proximidades das fontes acústicas, através da solução numérica da equação da onda dada pela equação (1). O ambiente marinho considerado, é um ambiente simplificado, considerando-se que as fontes se encontram em até 1000 metros de distância, sem variação de temperatura e salinidade. Desta forma, a velocidade de propagação é constante em todo domínio. O campo de pressão gerado pela simulação do modelo corresponde ao resultado da utilização de uma ou mais fontes com assinaturas acústicas de sete classes de navios encontrados no trabalho de McKenna et al. (2012). Para a solução numérica do modelo matemático, foi utilizado o Método de Diferenças Finitas - MDF. O MDF é uma das várias técnicas aplicadas para a soluções numérica de equações diferenciais. Seu princípio básico consiste na discretização do domínio contínuo em um conjunto de pontos finitos chamados de malha, ou grid. O método calcula uma solução numérica aproximada nos pontos do grid (BURDEN \& FAIRES, 2007).

As simulações feitas neste trabalho consideram uma associação das condições de contorno reflexivas e não reflexivas. A superfície do mar foi considerada refletora e para esta finalidade, na borda superior as condições de contorno utilizadas foram as reflexivas (ou de Dirichlet) onde os valores de pressão e fluxo no contorno são nulos, considerando a total reflexão da onda. Já nos contornos lateral direito, lateral esquerdo e inferior (fundo do mar), as condições adotadas são as não reflexivas. Essas condições foram adotadas por se considerar um ambiente marinho extenso de forma que no limite das bordas as ondas sejam atenuadas e não hajam reflexões. Os detalhes sobre as condições descritas acima podem ser encontrados em (SHAIKH \& HUANG, 2014). A simulação da propagação da onda acústica foi realizada considerando-se um ambiente submarino sintético com dimensões de 2000 metros de comprimento e 500 metros de profundidade. O objetivo é simular o nível de pressão acústica, considerando-se uma determinada quantidade de fontes sonoras dentro dessa região. O critério adotado para a escolha dessas dimensões objetiva cobrir uma região com até 1000 metros de raio com relação às fontes, visto que, estas dimensões estão relacionadas às faixas de distâncias críticas que podem causar efeitos como TTS e PTS, além de outros efeitos como estresse, mudança 
de comportamento, colisão com embarcações, encalhes, e possíveis danos fisiológicos (ERBE \& FARMER, 2000). A Figura 1 ilustra as etapas de solução do problema.

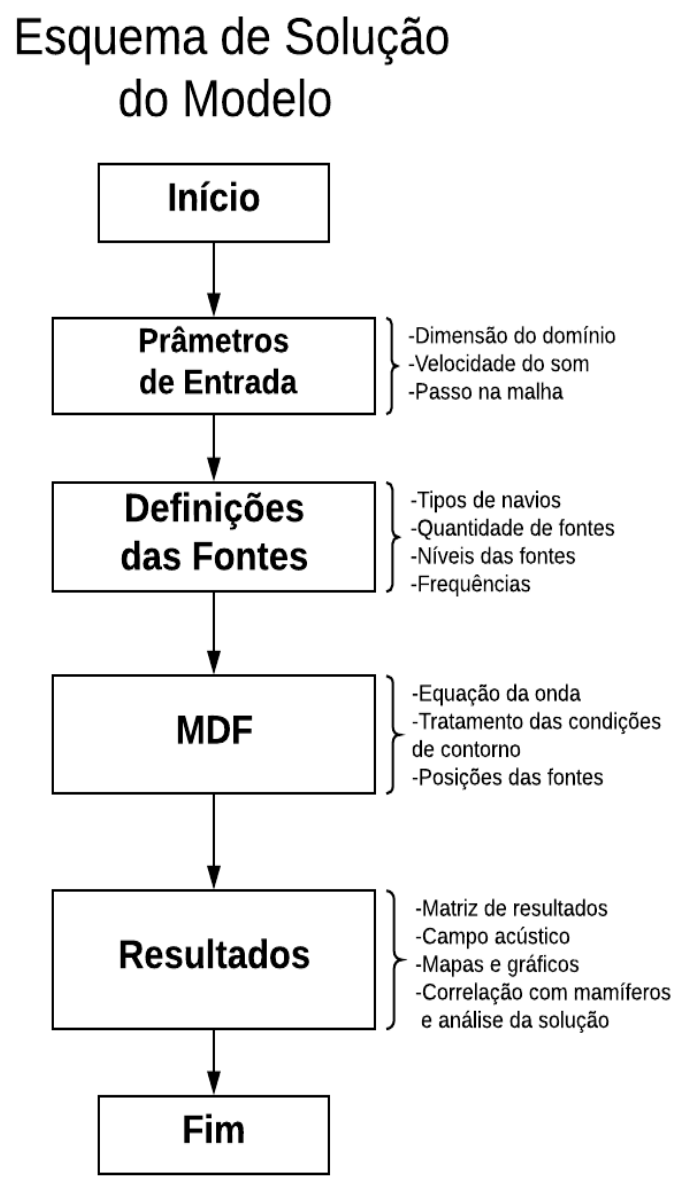

Figura 1. Esquema de Solução do Modelo.

\section{RESULTADOS E DISCUSSÕES}

Os resultados das simulações são apresentados em gráficos que apresentam cenários acústicos gerados pelas fontes que permitem analisar seus possíveis impactos nos mamíferos marinhos da subordem do Misticetos. As simulações foram realizadas considerando as fontes acústicas das sete classes de navios listadas na Tabela 1, com seus níveis de pressão associados a respectiva frequência $f_{1}=32, f_{2}=64, f_{3}=128$, $f_{4}=256, f_{5}=512$ e $f_{6}=1028$. Uma vez determinadas as frequências, é possível definir as frequências angulares $\omega_{1} \ldots \omega_{6}$. A representação de cada fonte é obtida pela equação (3), composta pelas frequências e níveis de pressão acústica de cada classe de navio considerada. Em todas as simulações a velocidade de propagação adotada foi de 1500 $\mathrm{m} / \mathrm{s}$ por considerar-se um meio homogêneo, sem variação de temperatura e densidade. 
Os resultados são gráficos representativos do nível de pressão acústica na região considerada, as secções transversais que representam a distribuição dos níveis acústicos ao longo da distância em uma determinada profundidade e os espectros dos sinais que relacionam as frequências e intensidades das fontes com as frequências e intensidades dos animais marinhos.

Para a primeira simulação, foi selecionada apenas uma classe de navio como sinal de fonte, correspondente a que apresenta os menores valores e variação de pressão acústica. O objetivo é observar o comportamento da propagação acústica, assim como a sensibilidade do sinal às baleias consideradas neste trabalho. Assim, o navio de classe Navio-taque de Produtos será considerado, com suas amplitudes: $A_{1}=174, A_{2}=176$, $A_{3}=175, A_{4}=169, A_{5}=170$ e $A_{6}=165$. O campo acústico resultante é representado na Figura 2. A fonte foi posicionada considerando-se suas coordenadas na malha em relação à origem, a 1000 metros de distância, com 5 metros de profundidade.

Deste modo, é possível obter-se uma ideia geométrica da distância em que um animal marinho pode circular em torno das fontes, em segurança. A simulação mostrou variações de pressão acústica na faixa de 60 a 180 dB. Estes níveis sonoros são suficientes para a ocorrência de danos físicos ou estresse nos animais, observando-se que estes devem estar a distâncias próximas de 200, 450 e 1000 metros para que ocorram efeitos como TTS ou PTS (a depender do tempo de exposição), entre outros efeitos que podem ser prejudiciais a esses animais.

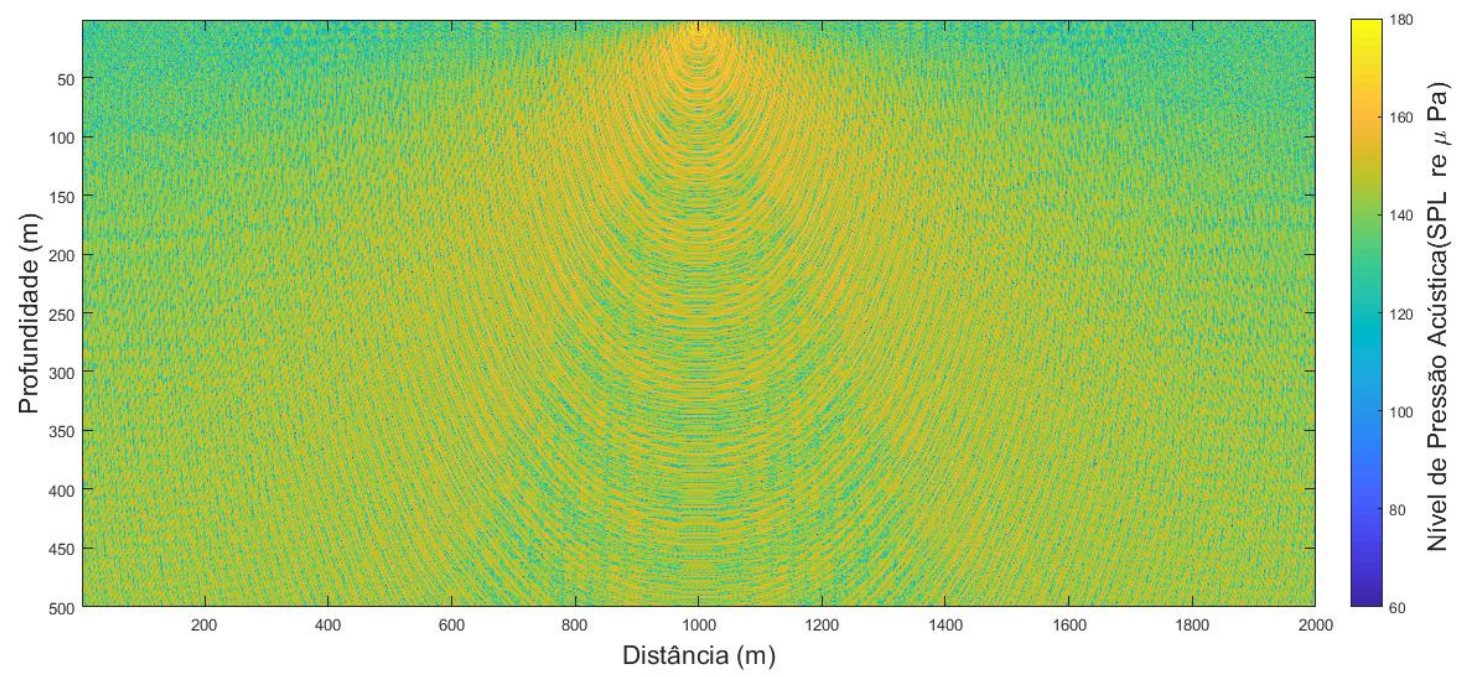

Figura 2. Representação do Campo Acústico para a Simulação dos Níveis de Ruídos Gerados pelo Navio de Classe Navio-taque de Produtos. 


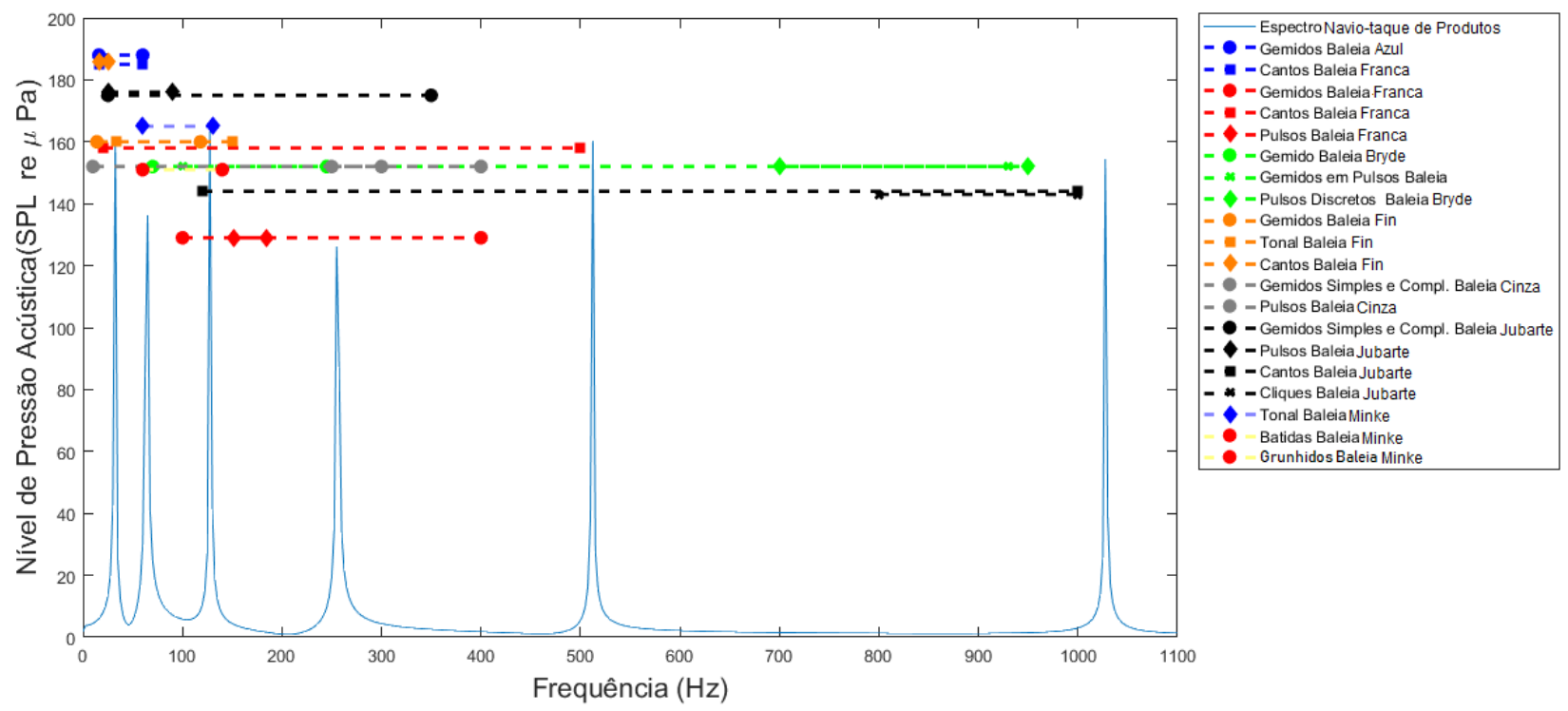

Figura 3. Representação do Campo Acústico para a Simulação dos Níveis de Ruídos Gerados pelo Navio de Classe Navio-taque de Produtos - adaptado de MCKENNA et al., 2012.

A Figura 3 mostra o espectro do sinal da fonte utilizada nesta simulação. Este espectro indica a relação dos níveis de pressão acústica com suas respectivas frequências, e é obtido aplicando-se a Transformada de Fourier, dada pela equação no sinal da fonte (BEERENDS, 2003). Assim, é possível correlacionar o espectro com as frequências e potências de vocalização dos Misticetos. Por exemplo, segundo dados da Tabela 2, a Baleia Franca (retas em vermelho) emite seus gemidos com faixa de frequência dominante de 100 a $400 \mathrm{~Hz}$ na potência 129 até $178 \mathrm{~dB}$. Caso uma baleia dessa espécie esteja circulando na região considerada por esta simulação, o sinal da fonte poderia interferir em seu gemido nas frequências 128 e $256 \mathrm{~Hz}$ caso essa baleia emitisse um gemido com $129 d B$ de potência. Analogamente, o espectro deste sinal faz interferência com as faixas de limites de frequências de vocalizações de todas as baleias exceto os sinais dos gemidos, pulsos e cliques da Baleia Jubarte, gemidos e canto da Baleia Azul e canto da Baleia Fin. Na simulação seguinte, foram consideradas as fontes de todas as classes de navios e suas respectivas variações de pressão acústica em banda de uma-oitava. Desta forma pode-se avaliar a propagação das fontes e como os sinais podem ser sensíveis apenas a uma única espécie de baleia. O critério para a seleção desta espécie para ser avaliada na simulação, consiste na escolha da espécie que apresenta a maior variação de vocalizações. Neste caso, a baleia que melhor se enquadra nesse critério é a Baleia Jubarte. A Figura 4 representa o campo acústico da simulação de propagação da fonte com as características acústicas de todas as classes 
de navios. Já a Figura 5 representa o espectro dos sinais desses navios assim como as faixas de vocalização da Baleia Jubarte (cantos com frequências 128 e 512 Hz). Neste caso, observa-se que se houver um aumento de 5 ou $10 \mathrm{~dB}$, os sons se tornam mais suscetíveis de interferir nos gemidos e pulsos da baleia. Nota-se também que as frequências do clique desta baleia não são afetadas devido à pouca variação de frequência do sinal que, neste caso não apresenta valores consideráveis (frequências de 500 a $1000 \mathrm{~Hz}$ ). Isso mostra que quanto maior a variação de frequência que um sinal pode emitir, maior será a probabilidade de se tornar sensível às vocalizações das baleias.

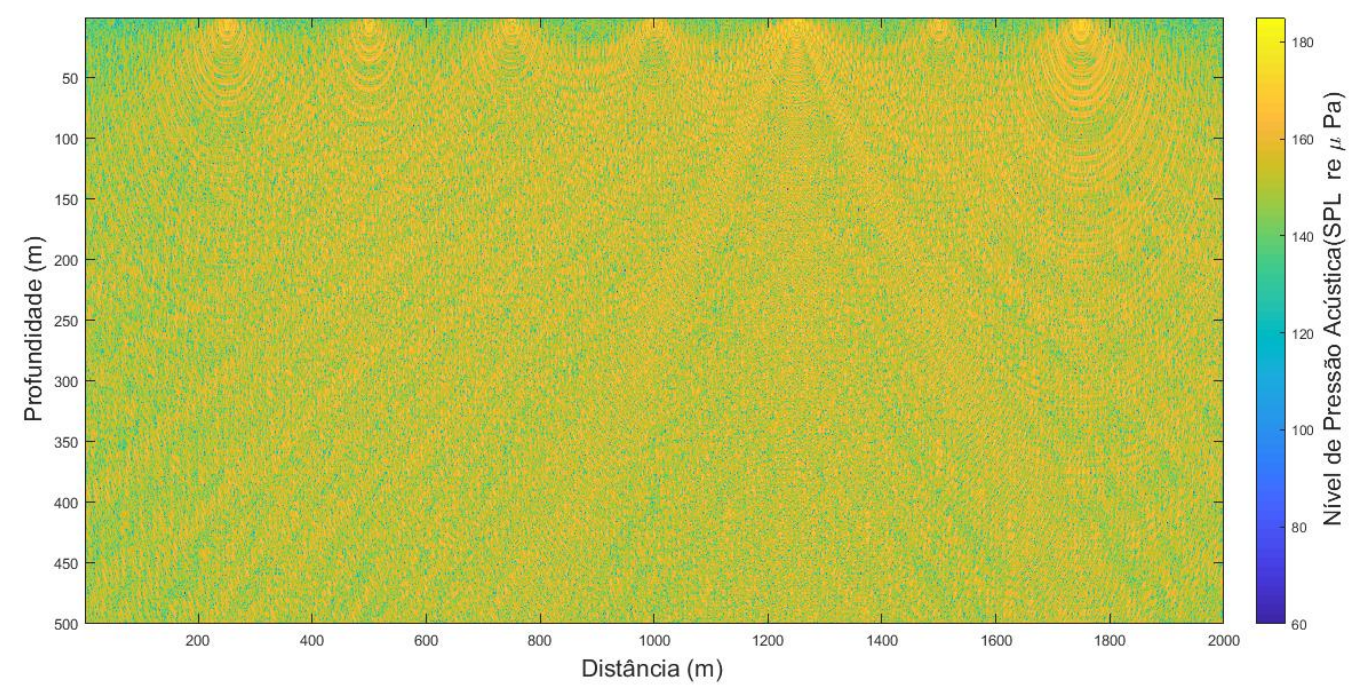

Figura 4. Campo Acústico da Simulação dos Níveis de Propagação de Ruídos Gerados por Todas as Classes de Navios.
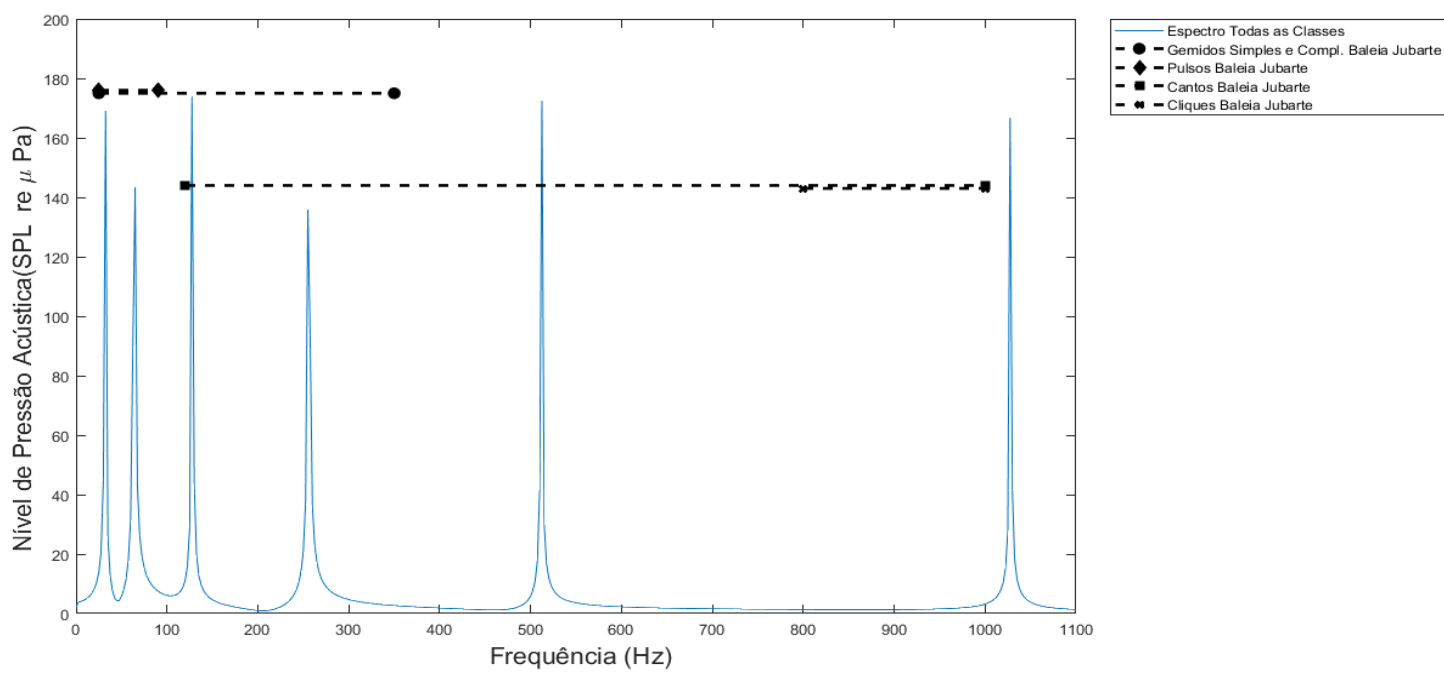

Figura 5. Espectro dos Sinais das Fontes de Todas as Classes de Navios e Faixas de Frequências Sensíveis as Vocalizações da Baleia Jubarte - adaptado de MCKENNA et al., 2012. 
DOI: $10.18605 / 2175-7275 /$ cereus.v12n2p104-118

Finalmente, foi realizada uma simulação considerando as fontes de todas as classes de navios listadas na Tabela 1, e supondo-se que esses navios possam emitir sinais que contém exatamente as frequências de vocalização das baleias listadas na Tabela 2. A principal diferença entre as demais simulações está no espectro dos sinais.

A Figura 6 ilustra o espectro dos sinais. Observa-se que a simulação reproduziu exatamente as faixas de frequências sensíveis aos animais marinhos, e demonstra que quanto maior a variação de frequências emitidas pelas fontes, maior será a probabilidade de afetar a vida desses animais. Mostra também que estes sinais podem causar maiores interferências em espécies de Misticetos que vocalizam com frequências menores que $500 \mathrm{~Hz}$.

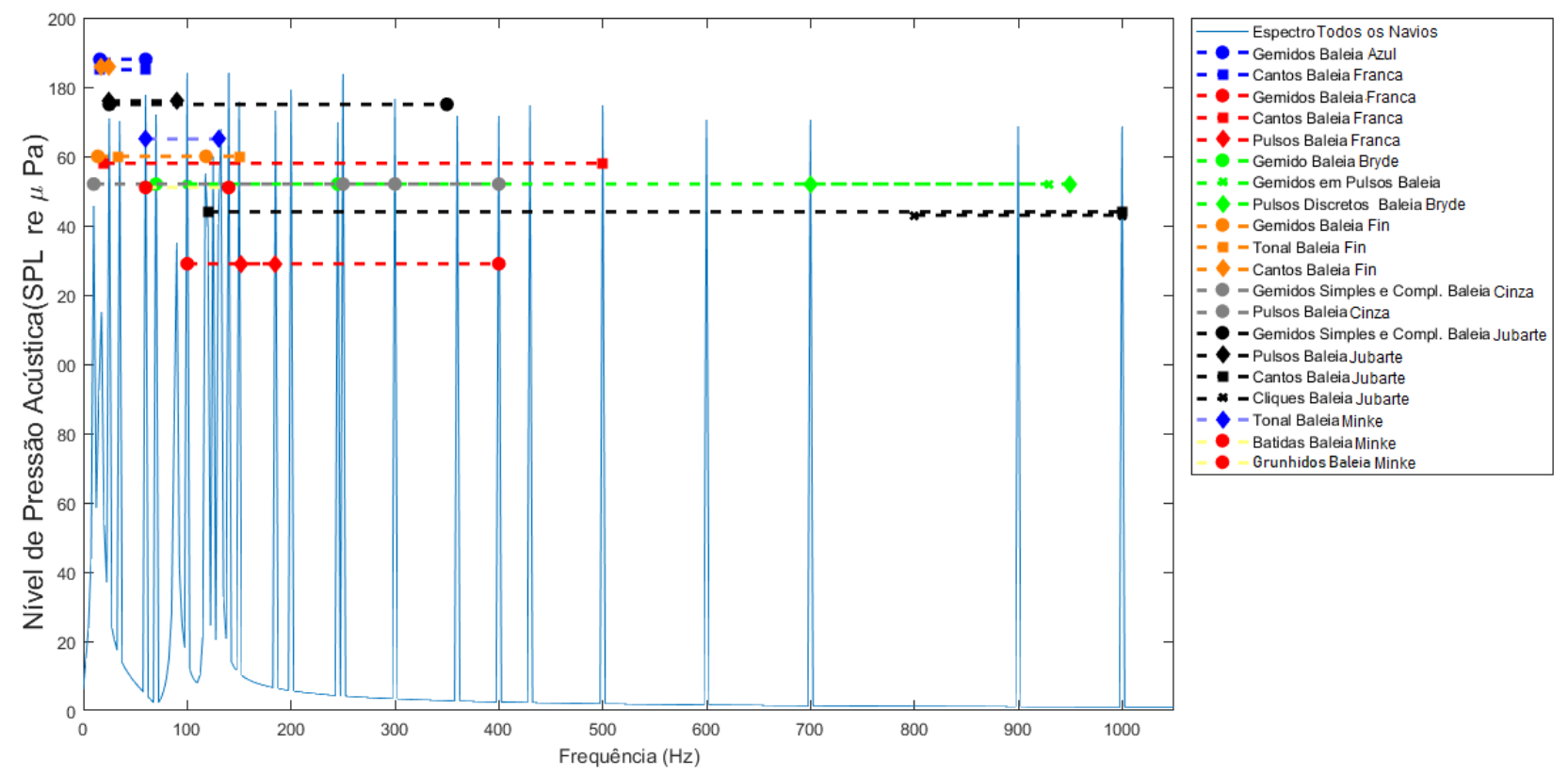

Figura 6. Espectro dos Sinais das Fontes dos Navios e Faixas de Frequência de Vocalização dos Misticetos Considerados neste Trabalho - Adaptado de MCKENNA et al., 2012.

\section{CONSIDERACÕES FINAIS}

No presente trabalho foi apresentada a modelagem matemática da propagação da onda acústica em duas dimensões com objetivo de avaliar os possíveis risco e impactos das ações provenientes do homem na natureza, em particular na vida de algumas espécies de mamíferos marinhos. Visto que as ações antropogênicas são capazes de produzir poluição sonora subaquática, o estudo foi voltado principalmente a analisar os possíveis impactos na vida desses animais que são extremamente dependentes do som. 
Através do método de diferenças finitas, foi possível solucionar as equações provenientes de um modelo, construído a partir da equação da onda. Tal solução, foi tomada em uma região sintética, capaz de posicionar as fontes sonoras e assim simular sua propagação acústica. Desta forma, a partir dos resultados gerados pelas simulações e considerando-se os dados característicos das fontes acústicas e das vocalizações de algumas espécies de Misticetos, foi possível verificar o quanto essas fontes podem influenciar a vida desses animais, do ponto de vista acústico.

O possível aumento dos ruídos nos oceanos, sobretudo os ruídos gerados pelas atividades antropogênicas, com a evolução das atividades produtivas, justificam o desenvolvimento de pesquisas científicas voltadas para estratégias de controle da poluição sonora nos oceanos e seu impacto na natureza. O presente trabalho está em sintonia com tais objetivos.

\section{AGRADECIMENTOS}

O presente trabalho foi realizado com apoio da Coordenação de Aperfeiçoamento de Pessoal de Nível Superior - Brasil (CAPES) - Código de Financiamento 001.

\section{REFERÉNCIAS}

AU, W. W. \& HASTINGS, M. C. Principles of marine bioacoustics. 1st ed., SpringerVerlag, New York, 2008.

BEERENDS, R. J., MORRCHE, H. G., VAN DEN BERG, J. C. and VAN DE VIRE, E. M. Fourier and Laplace transforms. 1st ed., Cambridge, 2003.

BURDEN, R. L. \& FAIRES, J. D. Análise numérica. 8ª ed., Cengage Learning, 2007.

ERBE, C. Underwater acoustics: noise and the effects on marine mammals. A pocket handbook. 3rd ed., JASCO Appl Sci Editor, 2011.

ERBE, C. \& FARMER, D. M. Zones of Impact Around Icebreakers Affecting Beluga Whales in the Beaufort Sea. The Journal of the Acoustical Society of America. n. 3, v. 108, p. 1332-1340, 2000.

IÓRIO, V. EDP um curso de graduação. Coleção matemática universitária. 3ª Edição, Instituto de Matemática Pura e Aplicada, 2012.

JEFFERSON, T. A., LEATHERWOOD, S.,WEBBER, M. A. Marine mammals of the world. 2nd ed., Academic Press, 2015. 
JENSEN, F. B., KUPERMAN, W. A., PORTER, M. B., SCHMIDT, H. Computational Ocean Acoustics. 2nd ed. Springer, New York, 2011.

JESUS, S. M., SOARES, C., ZABEL, F., SILVA, A. Marine strategy framework directive. Relatório da Universidade do Algarve e Marsensing, 2010. Disponível em: http://www.marsensing.com/pdf/pubs/MSFD_desc11_v4.pdf. Consulta: 23/03/19.

MCKENNA, M. F., ROSS, D., WIGGINS, S. M., HILDEBRAND, J. A. Underwater radiated noise from modern commercial ships. Journal of the Acoustical Society of America, v. 131, n. 1, p. 92-103, 2012.

REDONDO, L. \& MATEO, A. R. Ruido Subacuático: Fundamentos, Fuentes, Cálculo y Umbrales de Contaminación Ambiental. Ingeniería Civil. v .186, p. 73-94, 2017.

RICHARDSON, W.J. \& WÜRSIG, B. Significance of Responses and Noise Impacts. Marine Mammals and Noise. p. 387-424, 1995.

SANTOS, M. E., COUTINHO, M. N., RITA L. A. and GONÇALVES, E. J. Monitoring Underwater Explosions in the Habitat of Resident Bottlenose Dolphins. The Journal of the Acoustical Society of America. n. 6, v. 128, p. 3805-3808, 2010.

SHAIKH, S. \& HUANG, Y. W. Influences Analysis of Environmental Parameters on Sound Propagation in Shallow Water. Applied Mechanics and Materials. v. 556, p. 4815-4819, 2014.

WALSH, W. A. \& KOBAYASHI, D. R. A description of the relationships between marine mammals and the Hawaii-based longline fishery from 1994 to 2003. Report prepared by the University of Hawaii and Pacific Islands Fisheries Science Center, 2004. 